\title{
Understanding the Mental Health Literacy in Kurdish Refugees
}

\author{
Zibaei A* \\ PhD candidate, Manchester Metropolitan University, UK
}

*Corresponding author: Afrouz Zibaei, PhD Candidate, Manchester Metropolitan University, UK, Tel: +358440780071; Email: afrouz.zibaei@stu.mmu.ac.uk

\section{Research Article}

Volume 2 Issue 2

Received Date: July 02, 2018

Published Date: July 31, 2018

DOI: $10.23880 / \mathrm{mhrij}-16000124$

\section{Abstract}

Background: Health literacy is an important issue which is closely connected to health promotion, protection and disease prevention. The aim of this research is to study and raise awareness of mental health issues amongst Kurdish refugees in Finland. The methods and materials include reading and analysing previous studies conducted on this subject. The results indicate that Kurdish refugees suffer from multiple mental health conditions which should be taken into further consideration.

Keywords: Mental Health Disorder; Health Literacy; Kurdish Mental Health

\section{Introduction}

Health literacy is the degree of which an individual can understand and process health related information to make appropriate decisions. According to Kutcher and Wei (2016) [1] 'mental health literacy (MHL) is a construct that has arisen from the domain of health literacy' [1]. The term mental health literacy (MHL) was introduced by Jorm and colleagues as an extension of the concept of health literacy. It may be defined as awareness, knowledge and good opinion about mental disorders which help assessment, handling or prevention [2]. Mental health literacy is the ability to recognize mental health disorders and understand how to search appropriate mental health related information.

\section{Mental Health Disorders}

Mental health disorders are brain disorders which are an optimal level of thinking, feeling, and relating to others. Mental health disorders are many different conditions which are recognized as mental illnesses. According to THL (2012) [3] many mental and physical problems, as well as physical disabilities, are untreated amongst immigrants and refugees. Information research appears to lack awareness of the complex needs of immigrants and refugees.

\section{Refugees and Mental Health Disorders}

Throughout history, millions of people have been moved from one place to another place because of war, a political problem, and persecution by their own government, religious or ethnic fervour. According to research studies, migration and refugee's process is a stressful process, because they are at risk of culture shock and mental health problems [4]. Has explained that "most of us understand that in real life, immigrants experience difficulties when arriving a new country, difficulties like getting used to another climate, new environment, and different social or cultural customs." Weaver (1994) [5] has been categorized culture shock into three basic actions such as: I) Loss of familiar guides II) Breakdown of interpersonal communications III) Identity crisis. Migration and refugees culture shock have consequences such as Psychological irritation, physical stress, and Cultural Consumption. Research has indicated that 


\section{Mental Health \& Human Resilience International Journal}

refugees are one of the most vulnerable groups in our society in terms of risk for poor mental health [6]. The mental health disorders have different background among immigrants and refugees' groups. Beiser (2005) [7] has explained that the health of migrants and refugees have specific challenges in mental health issue includes; communication, cultural of symptoms and illness behavior, family structure has an effect on process acculturation and intergenerational conflict or impedance of adaptation and social integration. The mental health problems present in physical problems untreated, which can lead to mental disorders. Research studies suggest the cultural and religious beliefs about the causes and cures of mental health need to take into account as great influence the diagnoses and treatment process $[8,9]$.

\section{Kurdish Refugees and Mental Health Disorders}

Kurdish refugees who are living in Finland are suffering from different mental health disorders, according to the researchers of National Institute of Health and Welfare (THL) in Finland. They are suffering from psychological and physical health problems, such as emotional (sadness, grief, fear) [10] cognitive (loss of control, impuissance, worry, boredom, and hopelessness), physical (fatigue, sleeping problem, loss of appetite) and social and behavioral problems such as withdrawal symptoms, aggression and interpersonal difficulties are also common [11,12]. All Kurdish refugees may experience feelings of grief due to separation from their culture, society, family, friend and social connections, lack of employment, possessions and wealth, and loss of the ability to communicate, integrate and form a sense of identify and language barriers [13]. The Kurdish refugees are particularly vulnerable to disorders of both physical health and mental health. Kurdish refugees have a significant challenge in mental health issue and willingness to access health care and health information. There are different research studies have been conducted into the subject of Kurdish refugees and mental health. In 2010 Gülșen, et al. [14] conducted a study to investigate the Impact of forced Migration on mental health. Their 'study conducted with migrated Kurdish women both in the European Union (EU) and in Turkey aimed to explore the relationships between posttraumatic stress reactions, forced migration, and mental health status [14]. In other study Fatahi (2014) [15] has shown that how premigration, migration and post-migration factors have an impact on the mental well-being of Kurdish refugees settled in Sweden. This research study shows the highlight of anxiety and psychological problems among migrants in this country. After that in (2015) Rask, et al. [16] 'demonstrated a bidirectional relationship between physical function and depression.' They made conclusion that 'Mental health symptoms are significantly associated with mobility limitation both in the studied migrant populations and in the general Finnish population [16]. In 2017 Ibrahim and Hassan have considered PTSD symptoms with torture and other traumatic events among Syrian Kurdish refugees who are living in Arbat camp in Iraq [17]. This research has reported that Kurdish men traumatic experiences more than Kurdish women. They have claimed that 'the war trauma, torture and postmigration of refugees are affecting to develop mental health disorders.' According of these studies commune mental disorders among Kurdish refugee population are including Post-Traumatic Stress Disorder (PTSD), Depression and Anxiety. However, there are limited researches on the cause of mental health disorders and Kurdish refugees. In this study other aspects of the issue include Pain Disorder, Anger and Aggression, Alcohol Dependence, Obsessive-Compulsive Disorder were referenced. Language, cultural barriers and lack of understanding about health care system, most of the Kurdish immigrants have communication and language problems and they use their family member as interpretation. Unfortunately, the children cannot be good interpretation because of their age and lack of medical information. Most of the Kurdish women use their husbands as a translator to visit the doctor. The translation of family members is not enough to diagnose health problems. That makes treatments hard and complicated. Because of shame and fear, they are not aware of the medical terminology and cultural differences. Medical problems are often diagnosed in the later stage and most of them are continuing. Because of language barrier and health information, the gap between the doctor and the Kurdish immigrants and refugees are wide. According to multiple research studies, acculturation decreases the risk of psychological distress. Communicating health information is more difficult today than ever.

\section{Methods and Materials}

The studies were based on personal experience and education and the combination of relevant research study which are available on the topic of refugee mental health issue. The PubMed, PubMed Health, MEDLINE, PsycINFO (EBSCO), SAGA, Elsevier, and WHO website were used.

\section{Results and Conclusion}

\section{Posttraumatic Stress Disorder (PTSD)}

PTSD is a disorder that develops in some people who have seen or lived through a shocking, scary, or dangerous event. Higher prevalence of trauma exposure is 


\section{Mental Health \& Human Resilience International Journal}

related to higher levels of psychopathology, especially anxiety and depressive disorders, psychological distress, weak character, nervous breakdown, serious medical condition, stress, homesickness, and physical condition (e.g. a migraine or back pain). Psychosocial issues of PTSD are often related to life circumstance, family relations, poor education and poor health. According to the 2012 research conducted by Deville et al explanation posttraumatic stress disorder (PTSD) is a significant health problem among migration and refugees in the worldwide [18]. Most of the Kurdish refugees have experienced a series of different events, Kurdish mental health consequences have been described after severe traumatic events in their background. Immigration and refugee is always a big life change for Kurdish people too. Because immigration and asylum seeker is a psychologically heavy process. Trauma often has related to sleeping disorders, memory disorders, and attention problems. Trauma has an impact on the ability to learn and as well as difficult to learn the language or work. Research about refugee and immigrant has done in 2010-2012 among 3000 Russian, Somali and Kurdish adult health and well-being in six cities in Finland. Research studies were 1000 person per language group. Research result has shown that $90 \%$ of Kurdish refugee and immigrant are suffering from PTSD.

\section{Post-migration Stressors}

Many refugees are suffering from a host of traumas and mental illnesses. They have psychological problems, including post-traumatic stress disorder and depression. Kurdish refugees have been exposed to multiple traumas making them lead to mental health problems later. The Disorders of Extreme Stress (DESNOS) syndrome shares several symptoms with the formal PTSD diagnosis, but also expands it to include several important symptoms such as alterations in self-perception, inter-personal impairment, self-destructive behaviors and somatization [19]. Trauma and Disorders of Extreme Stress (DESNOS) including dissociative disorders, continue to be grossly under diagnosed [20]. Post-migration stressors have negatively affected mental and physical health situation. Refugees have personal stories of experienced or witnessed war, violence, targeted persecution, forced work, forced migration and family separation. Unfortunately, many refugees have experiences of poverty, interracial conflict, family instability and parental psychosocial distress [21]. They arrive at the new countries with limited education, low literacy, and language problems. The learning process is difficult and slow because research studies have explored that there is a relationship between trauma and learning process. Researchers have suggested that refugees need to be grounded on a pedagogical framework [22].

\section{Pain and Mental Health Disorder}

According to Harvard medical school explanation pain and chronic pain is an emotional and physical condition, therefore pain is depressing and depression increase pain. People with chronic pain have been characterized by abnormalities in brain hormones, low energy, mood disorders, muscle pain, and impaired mental and physical performance. Psychological pains can descriptions such as mental pain, emotional pain, psychic pain, social pain, spiritual or soul pain and suffering pain. In (2006) Parlee [23] has explanted that researcher's intent to observation relationship between mental illness and chronic pain, and interaction biological, psychological and social influences. For example, chronic pain is experiences of refugee's men and women as a cause of emotional, depression and altered personalities, which all had high consequences on their social interactions, give rise to change and loss of social relations. Chronic pain has a severe negative impact on quality of life, for example, psychosomatic disorders such back pain, headache, body pain, dyspepsia, abdominal pain, and fatigue and muscle pain. Kurdish refugees have been left their home countries because of war or persecution and politic problems. Unfortunately, Kurdish refugees have experienced a lot of difficult things already in their home countries. For many, the changes have been perhaps painless. The refugees have reported feelings of loneliness, homesickness, fear, and confusion in new culture and country. According to research studies about refugees and immigrants in Finland in 2012, 30\% men and $60 \%$ women suffering from a different kind of pain. This research has bested on 3000 Russian, Somali and Kurdish adult health and well-being.

\section{Anger and Aggression among Refugees}

Deffenbacher and McKay (2000) have explained that anger is a normal emotional is experienced by everyone. However, anger like other feelings is controlled by emotional, physiological and cognitive characteristic. Research studies in this filed explored that many young refugees experience social, emotional and behavioral problems connected with their pre- and post-migration stressful experiences. Impacting needs have been identified in the areas of loss and trauma, cultural transfer, housing, education, employment, family support and structure, general health and wellbeing access to health services. Many Kurdish refugees and migrants have experienced of traumatic events includes: evidencing family murdered or danger, amputation, Loss of home and family, forced a child soldier, hunger and prison.

Historical, religious, ethnic and social dynamics all contribute to shaping. Kurdish cultural concepts of the 


\section{Mental Health \& Human Resilience International Journal}

person influence how people experience and express suffering, how they explain illness and misfortune, and how they seek to help [24]. Anger is emotional mood that surfaces the most easily and be expressed as aggressive behavior, both within the family and outside of it. Kurdish refugees' men don't cry and are not afraid or sad, controlling this with weakness [25].

\section{Obsessive-Compulsive Personality Disorder}

Obsessive-compulsive personality disorder (OCPD) is a mental disorder condition that the person involved with their rules, orderliness, and control. This mental disorder can affect both men and women. The exact cause of OCPD is unknown. Obsessive-compulsive personality disorder causes interpersonal problems with family members, friends, example actions like excessive hand-washing or repeatedly checking the locks on windows and doors. They have obsessively clean and hygienic. Obsessivecompulsive personality disorder people have problem delegating, trusting other people, sharing responsibilities or compromising. Research studies explored that people who are suffering from personality disorders are often also diagnosed by symptoms of depression. However, there is no evidence indicating that relation between refugee process and Obsessive-Compulsive Disorder.

\section{Depression and Anxiety}

Among older adults of Kurdish refugees and immigrants a higher proportion who are living in Finland, have at least one of these seven chronic conditions: asthma, cancer, heart disease, diabetes, high blood pressure, obesity, or anxiety and depression. These rank among the costliest medical conditions in Finland [26]. Kurdish refugees who come to Finland have many different reasons. Immigrant and refugees (include Kurdish people) have little knowledge of the Finnish society and language. So, they face the risk of depression due to their lack of participation in the society [27]. According to research studies, immigrant and refugee populations often experience extensive post migration stressors, including distressing life events and poor physical health condition. Research shows that stressful life events experienced by older residents in a new country, such as financial crisis and separation from someone close family and friend, contribute to depression $[28,29]$.

\section{Alcohol Dependence}

Refugee process of acculturation, moving from one culture to another, has an impact on drinking levels and the way in which alcohol is viewed by the community. The impact of immigration on the use of alcohol has been found to be influential by previous researchers. Also, report drinking amongst women is far lower than among men in minority groups who have left their countries of origin [30]. These findings also corroborate evidence of discrimination amongst immigrant groups and how this correlates with heavy drinking [31]. Research Studies that explored the impact of drinking on physical health, mental health, and family relationships. Stories about alcohol improving circulation, blood, and kidney function were identified and the idea that alcohol works better than antidepressants for lifting the mood. Dependent on alcohol is associated with social problems and mental health difficulties. Alcohol has also been found to play a fundamental part in all crime, and crimes of violence in particular [32]. According to research studies in Finland 2012 based in refugee and immigrant studied 3000 Russian, Somali and Kurdish adult health and well-being. Research studies [3] have shown that Kurdish refugees' women use $20 \%$ and men use $60 \%$ alcohol in Finland.

\section{Discussion}

There are also many other different conditions that are recognized as mental illnesses among Kurdish refugee. They are including Suicide Prevention, Sleeping Disorders. Social Phobia, Borderline Personality Disorder (Paranoid, Schizoid, Antisocial, Borderline, Narcissistic, Historic, Dependent personality, demanding) There are also different aspects of the issue which are not referenced: Homesickness, Relational Disorder, Irrationality, Behavioral Disorder, Factitious Disorder, Schizophasia (word salad, Thought disorder (TD)), Kleptomania. Pathological gambling, Mythomania, and Pathological lying.

Community Health Improvement Plan (2015) [33] has been explained that people from different cultural backgrounds, health literacy is influenced by belief systems, communication skill, and understanding and reaction to health information knowledge. Mental health literacy gives the knowledge to prevention and exposure to treatment; at the same time, it keeps them knowledgeable of the modern health facilities available to them to promote their quality of health. Mental health literacy and mental health information can help to prevent health.

\section{References}

1. Kutcher S, Wei Y, Coniglio C (2016) Mental Health Literacy: Past, Present, and Future. Can J Psychiatry 61(3): 154-158. 


\section{Mental Health \& Human Resilience International Journal}

2. Jorm AF, Korten A, Jacomb P, Christensen H, Rodgers B, et al. (1997) Mental health literacy. A survey of the public's ability to recognize mental disorders and their beliefs about the effectiveness of treatment. Med J Aust 166(4): 182-186.

3. Castaneda AE (2012) Immigrants' health and welfare research report results (Maamu THL 2012).

4. Madelaine H (2009) Translating Pain: Immigrant Suffering in Literature and Culture. University of Toronto Press Incorporated, Toronto Buffalo London.

5. Weaver GR (1994) Understanding and coping with cross-cultural adjustment stress. In: GR Weaver (Eds.), Culture, communication and conflict: Readings in intercultural relations, Ginn Press, Needham Heights, pp: 169-189.

6. Fazel M, Wheeler J, Danesh J (2005) Prevalence of serious mental disorder in 7000 refugees resettled in western countries: A systematic review. Lancet 365(9467): 1309-1314.

7. Beiser M (2005) The health of immigrants and refugees in Canada. Can J Public Health 96(2): 30-44.

8. Lay B, Lauber C, Rossler W (2005) Are immigrants at a Disadvantage in Psychiatry in-patient care?. Acta Psychiatrica Schandinavica 111(5): 358-366.

9. McCrone P, Kamal deep B, Craig TKJ, Curtis S (2005) Mental Health Needs, Service Use and Costs among Somali Refugees in the UK. Acta Psychiatrica Schandinavica 111(5): 351-357.

10. Momartin S, Silove D, Manicavasagar V, Steel Z (2004) Complicated grief in Bosnian refugees: Associations with posttraumatic stress disorder and depression. Comprehensive Psychiatry 45(6): 475-482.

11. Vukcevic M, Dobric J, Puric D (2014) Psychological characteristics of asylum seekers from Syria. Survey of the mental health of asylum seekers in Serbia. UNHCR Serbia, Belgrade 73-85.

12. Acarturk C, Konuk E, Cetinkaya M, Senay I, Sijbrandij M, et al. (2015) EMDR for Syrian refugees with posttraumatic stress disorder symptoms: results of a pilot randomized controlled trial. Eur J Psychotraumatol 6: 27414.

13. Murray J, Janson CJ, Farrington DP (2007) Crime in adult offspring of prisoners: A cross-national comparison of two longitudinal samples. Criminal Justice Behavior 34(1): 133-149.

14. Gülşen C, Knipscheer J, Kleber R (2010) The Impact of Forced Migration on Mental Health: A Comparative Study on Posttraumatic Stress Among Internally Displaced and Externally Migrated Kurdish Women. Traumatology 16(4): 109-116.

15. Fatahi N (2014) The Impact of the Migration on Psychosocial Well-Being: A Study of Kurdish Refugees in Resettlement Country. Sahlgrenska Academy at Gothenburg University, Institute of clinical sciences Department of radiology Sahlgrenska University Hospital, Sweden. Journal of Community Medicine Health Education 4(2): 273.

16. Rask S, Castaneda AE, Koponen P, Sainio S, Stenholm S, et al. (2015) The association between mental health symptoms and mobility limitation among Russian, Somali and Kurdish migrants: a population-based study. BMC Public Health 15: 275.

17. Ibrahim H, Hassan QC (2017) Post-traumatic Stress Disorder Symptoms Resulting from Torture and Other Traumatic Events among Syrian Kurdish Refugees in Kurdistan Region, Iraq. Front Psychol 20(8): 241.

18. Devillé W, Lindert J, Grujovic G, Iodice J, Petrova R (2012) Refugees Worldwide. In: Elliott D \& Segal UA (eds.), Santa Barbara, Refugee Health, Refugees World, California: Praeger.

19. APA (2000) Diagnostic and statistical manual of mental disorders (4th ed., text revision). American Psychiatric Association, Washington, DC.

20. Axe G, Berkowitz R, Chinman G, Hall K, Leiberg G, et al. (1993) Dissociative disorders in psychiatric inpatients. Am J Psychiatry 150(7): 1037-1042.

21. Hyman I, Beiser M, Vu N (1996) The Mental Health of Refugee Children in Canada. Refuge 15(5): 4-8.

22. Freire M (1990) Refugees: ESL and Literacy Trying to Reinvent the Self in a New Language. Refuge 10(2): 36.

23. Parlee J (2006) Chronic Pain and Mental Illness - How to Manage.

24. Mekki-Berrada A (2010) Islam in Anthropology of Mental Health: Theory, Ethnography and Clinic of an 
Alternative Look. Münster-Hamburg-London- Wien: Lit Verlag, Studies of Social Anthropology.

25. IOM (2015) Self-help booklet for men in crisis, international Organization for Migration, Beirut, Lebanon.

26. Number B (2004) Cultural Competence in Health Care: Is it important for people with chronic conditions?. Georgetown Health Policy Institute 5.

27. Forsander A, Ekholm L, Hautaniemi P (2001) Encountering Ethnic Groups. Multiethnic Society and Work Helsinki University Press.

28. Gellis ZD, Taguchi A (2004) Depression and health status among community dwelling Japanese American elderly. Ciinicai Genmtoiogisl 27(3): 23-38.

29. Mui AC, Kang SY (2006) Acculturation stress and depression among Asian immigrant elders. Soc Work 51(3): 243-255.
30. Schiff M, Rahav G, Teichman M (2010) Israel 2000: Immigration and Gender Differences in Alcohol Consumption. The American Journal on Addictions 14(3): 234-247.

31. Kim I, Spencer MS (2011) Heavy drinking, perceived discrimination, and immigration status among Filipino Americans. Substance Use and Misuse 46(10): 1256-1264.

32. Single E, Beaubrun M, Mauffret M, Minoletti A, Moskalewicz J, et al. (1997) Public drinking, problems and prevention measures in twelve countries: results of the WHO project on public drinking. Contemporary Drug Problems 24(3): 425-448.

33. CHIP (2015) city of Hartford. Community Health Improvement Plan. 